\title{
Group Polarization Based on Agent Emotional Characteristics and Credibility
}

\author{
Shuyang Jiang and Hu Wang $\mathbb{D}$ \\ School of Economics and Management, Southeast University, Nanjing 211189, China \\ Correspondence should be addressed to Hu Wang; whseu94@163.com
}

Received 22 March 2021; Revised 30 June 2021; Accepted 14 August 2021; Published 20 August 2021

Academic Editor: Yue Song

Copyright (C) 2021 Shuyang Jiang and Hu Wang. This is an open access article distributed under the Creative Commons Attribution License, which permits unrestricted use, distribution, and reproduction in any medium, provided the original work is properly cited.

\begin{abstract}
The frequent occurrence of group polarization in many internet mass incidents produces adverse impacts on the social stability and poses a significant challenge for social management. We construct an opinion evolution model with agent emotional characteristics and credibility based on the Deffuant model to study the evolutionary mechanism of group polarization, which connects the agent's firmness with the agent's opinion, taking a full account of the agent heterogeneity. We analyze the impacts of initial opinion distribution, network structure, and opinion leader on the group opinion evolution. The results show that group polarization is easier to form when the initial opinions are in a normal distribution, and group polarization will also form under the impact of initial minority agents with extreme opinions. Different network structures will pose different effects on the group opinion evolution, and group polarization is easier to form in the small-world network and BA scale-free network. In addition, opinion leader also affects the group opinion evolution, and it will hinder the generation of group polarization.
\end{abstract}

\section{Introduction}

At the early stage of internet public events, certain opinion intensity will be generated in the group, and the initial opinion intensity will be strengthened after the interactions between agents in the group as time goes by, and the consistent opinion intensity will become more and more extreme $[1,2]$. Moreover, the progress of the internet and appearing of the virtual community also promote such opinions to develop in an extreme way. Since the agents in the network are more inclined to communicate with agents who have similar opinions to theirs than with those whose positions are opposed to or more different from theirs, agents will strengthen their initial opinions after communicating with those holding similar opinions with them [3]. Due to the collaborative filtering of the internet, agents in the network can only see information that is similar to their own opinions or with common preferences [4]. On the other hand, social network sites and platforms divide the internet users into groups by interest, career, and other characteristics, which aggravates the community socialization of "birds of a feather flock together," so that the internet users easily come into contact with agents who have similar views and positions with their own. Meanwhile, the anonymity of online socializing makes it possible to communicate with others easily, thus promoting the formation of opinion consensus [5]. Group consensus refers to the consistency of agent opinions in a group. To some extent, group polarization can be regarded as a special case of group consensus, i.e., agents show the same extreme views on events, and group polarization usually has a negative impact on social stability. Group polarization is essentially an irrational behavior in the network public opinion development [6]. Since the public generally vents their feelings by expressing their views and opinions on hot social events on the internet, such irrational behavior generally generates an extreme opinion, namely, network public opinion polarization. The canard of the internet, as the descendant of group polarization, develops with the formation of group polarization. Group polarization not only seriously disrupts the network order but also may promote violent aggregate behaviors, bringing a serious negative impact on social stability. 
As an important component of social physics, opinion dynamics aims to study the complex group behaviors that emerge from opinion interactions between local agents in the whole scope by defining the opinion states of agents and the basic process of the changes of such opinions [7]. The complex and counterintuitive behavior of social systems and their macrolevel collective dynamics can be better understood by means of complexity science. Therefore, the agent based model of complexity science has been widely used in the study of social group behavior [8]. At the early stage of the network public opinion, there are different opinions; with time going by, multiple opinions will gradually reach a consensus, thus forming a unified opinion intensity. Such group polarization shown in public opinion has aroused the wide concern of scholars. Scholars have proposed various opinion evolution models, including the Ising model [9]; the voter model $[10,11]$; the Galam majority regular model $[12,13]$; the Sznajd model $[14,15]$; the bounded confidence model [16, 17]; the extended opinion evolution model [18-23] based on the above models, which generally includes the following aspects when simulating the group behavior: opinion interval (binary opinion, multiple discrete opinion values, and continuous spectrum); network structure (regular network, random network, small-world network, scalefree network and weighted network); opinion update rules (agents will update their opinions under the impact of the decisions of other agents). Such opinion evolution models simplify the social system and assign different characteristics to agents to study the impact of agent characteristics on the development of social network public opinion, such as the impacts of agent opinion acceptance [24, 25], degree of bigotry [26], and memory effects [27] on the group opinion evolution. In reality, agents are independent but interact with and are affected by the whole social environment, so agent's opinion on an event will be influenced both by the intrinsic factors and the external factor (social environment) [28]. With the development of the complex network, interactions between agents, in reality, can be approximately abstracted as a complex network, which can be used to study the interaction of opinions between agents and the evolutionary process of the group behavior under different network structures to reveal the mechanism of social public opinion evolution [29, 30].

Previous studies on opinion evolution models focused on the simplification of actual situations. Namely, it homogenizes all agents or divides agents into groups based on different characteristics. However, in reality, the agent characteristics and relationships between agents are much more complex. In fact, each person has various agent characteristics, and different agents generate different relationships, so these models cannot reflect the group opinion evolution process in reality. Agents change their opinions according to the current opinions and emotions of the agent and others. In other words, the evolution of agent opinions is affected by various internal and external factors, so it is difficult to accurately describe the group opinion evolution through agent interactions [31]. In our daily life, communication is a way for people to pass on the information and express their attitudes. On the other hand, people will also unconsciously change their own emotional characteristics. For example, to achieve certain purposes, the media and opinion leaders always transmit related information through special expressions to cause emotional resonance of the public, thus influencing the evolution of the group behavior upon the emotional changes. Related studies have shown that emotion plays an important role in group polarization $[32,33]$. Agent's emotional characteristics can be reflected by the agent's attitude toward the event, while the change of the agent's opinion depends on his/her own emotional characteristics toward the event. Generally, the clearer an agent's attitude toward the event (namely, when the agent's opinion is in the extreme state, such as agree or disagree), the less likely he/ she is to be influenced by others and change his or her opinion. Conversely, the unclear or neutral an agent's attitude toward the event, the more likely he or she is to be influenced by others and change his/her opinion [34]. In addition, the interaction of opinions between different agents is also affected by the relationship between them. Generally, the closer the relationship between agents, the more conducive to the interaction of opinions.

The contributions of this paper are as follows. First, we connect the agent's firmness with the agent's opinions on the event to construct an opinion evolution model with agent emotional characteristics and credibility based on the Deffuant model, in which the agent heterogeneity is fully considered. Second, we analyze the impacts of different network structures on the group opinion evolution from the perspective of the complex network. Finally, we introduce the opinion leaders and analyze the role of opinion leaders in the group opinion evolution.

The structure of this paper is organized as follows. Section 2 introduces the methodology. Section 3 presents the results. Section 4 concludes the paper.

\section{Methodology}

2.1. Deffuant Model. The Deffuant model is a widely used bounded confidence opinion evolution model, which is an update model of the previous binary opinion model $[35,36]$. In this model, opinions are continuous values distributed in a defined spectrum, only the agents with similar opinions will communicate with each other, while those with great different opinions will not communicate and keep their original opinions. Meanwhile, the agents with similar opinions will promote the assimilation of their opinions. The Deffuant model can more accurately simulate the actual process of opinion interaction and information transmission. We assume that the opinions of two random agents $i$ and $j$ in the network at time $t$ are $o_{i}(t)$ and $o_{j}(t)$, the opinions at time $t+1$ are governed by the following equations: 


$$
\left|o_{i}(t)-o_{j}(t)\right| \leq d, o_{i}(t+1)=o_{i}(t)+\lambda\left(o_{j}(t)-o_{i}(t)\right), o_{i}(t+1)=o_{j}(t)+\lambda\left(o_{i}(t)-o_{j}(t)\right),
$$

$$
\begin{aligned}
& \text { If } \\
& \left|o_{i}(t)-o_{j}(t)\right|>d, o_{i}(t+1)=o_{i}(t), o_{j}(t+1)=o_{j}(t),
\end{aligned}
$$

where $d$ is the tolerance threshold, $\lambda$ is the convergence coefficient, $\lambda \in[0,1]$, indicating the degree of acceptance of other agents' opinions by the agent. $\lambda$ determines the convergence rate of the opinions in the group, so the higher the $\lambda$ is, the closer the updated opinion value of an agent is to the opinion value of another agent in the interaction. Generally, $\lambda$ is 0.5 , indicating that the updated opinion is the average of the opinions of the two agents who communicate with each other. However, in reality, each agent has different characteristics and degree of acceptance of other agents' opinions. Agents with the lowest or highest acceptance of opinions are generally minority, the vast majority of agents' acceptance of opinions is in the middle level. Therefore, in this paper, we will use the normal distribution to reflect the agent's degree of acceptance of opinions.

2.2. RA Model. RA model (The Relative Agreement Model) is an extended bounded confidence model [34]. It introduces the uncertainty of opinion into the opinion evolution process and considers that agent's opinion is not a certain value but a spectrum that extends from the opinion value as the center to both ends, and the agent's attitude can be expressed by this opinion spectrum. The changed opinion value of agent $i$ impacted by agent $j$ is related to the size of the overlap between the opinion intervals of two agents, while the opinion interval between the two agents also influences each other. Meanwhile, if the opinion intervals of the two agents are different, the interaction effect is not symmetric. According to the study, the agent with a small opinion interval is more influential. In reality, agents with higher self-confidence are easier to persuade the agents with lower self-confidence because agents with lower self-confidence have a larger opinion spectrum, with unclear opinions, and are easy to change their opinions under the impact of others, while the agents with higher self-confidence have relatively small opinion interval, with clear opinion, and are not susceptible to external influences to change their opinions.

\subsection{Construction of the Opinion Evolution Model Based on Agent Emotional Characteristics and Credibility.} According to the above studies on the evolution of the group behavior, the change of an agent's opinion is often affected by the change of emotion, while the agents with extreme opinions always have higher emotional characteristics and self-confidence, with a firm attitude, so they are less likely to be influenced by the external factors and change their opinions. The psychological research [37] also confirms that extremists are more resistant to externally generated than moderates, and extremity does not necessarily beget irrationality, supporting the above hypothesis. For example, as for some hot events, some agents are not interested in it or consider that these events are not related to themselves, they may not express an obvious attitude tendency or keep a neutral attitude toward these events, while some agents are interested in it or consider that these events are closely related to themselves, they may pay much attention to the development of these events and make comments with clear attitude tendency. These agents have strong emotional characteristics and firm attitudes to stick to their opinions and are not easily influenced by external factors and change their attitudes. We assume that the range of agent's opinion is $[0: 1]$. With the increase of the opinion value, the agent will have a clearer attitude. When the opinion value is 1 , the agent has a clear attitude tendency and emotional characteristics; when the opinion value is 0 , the agent has an equivocal attitude tendency, with a neutral attitude and lower emotional characteristics. Similarly, when the range of agent's opinion is $[-1: 0]$, the opinion value is -1 , indicating the agent is clearly opposed to the event, with strong emotional characteristics. In addition, credibility is also a fact that affects the interaction of opinions of different agents. In this paper, we construct an opinion evolution model with agent emotional characteristics and credibility on the basis of the bounded confidence model [38].

$$
\begin{aligned}
& \left|o_{i}(t)-o_{j}(t)\right| \leq R_{i j} d_{i}, o_{i}(t+1)=o_{i}(t)+\lambda\left(o_{j}(t)-o_{i}(t)\right), \\
& \left|o_{i}(t)-o_{j}(t)\right| \leq R_{i j} d_{j}, o_{i}(t+1)=o_{j}(t)+\lambda\left(o_{i}(t)-o_{j}(t)\right), \\
& \left|o_{i}(t)-o_{j}(t)\right|>R_{i j} d_{i}, o_{i}(t+1)=o_{i}(t), \\
& \left|o_{i}(t)-o_{j}(t)\right|>R_{i j} d_{j}, o_{j}(t+1)=o_{j}(t),
\end{aligned}
$$

where $R_{i j}$ indicates the credibility degree of agent $i$ to agent $j$, $R_{i j} \in[0,1]$. The credibility can be calculated by the equation: $R_{i j}=C_{i j}+1 / N_{i}$, where $C_{i j}$ indicates the number of common nodes of the two agents, $N_{i}$ indicates the number of nodes of the agent $i$. The larger the percentage of common nodes with agent $j$ on the connected nodes of agent $i$, the higher the credibility degree of agent $i$ to agent $j$. When $C_{i j}=N_{i}-1$ and $R_{i j}=1$, indicating the maximum of credibility degree of agent $i$ to agent $j . d_{i}=F\left(\left|o_{i}\right|\right)$ and $d_{j}=F\left(\left|o_{j}\right|\right)$ indicate the agent's tolerance threshold is a power function of its opinion value, and the tolerance threshold becomes smaller as the opinion value gets larger, this can be expressed by the following equation:

$$
d_{i}=d_{m}-\left|o_{i}\right|^{x}\left(d_{m}-d_{n}\right) \text {. }
$$

When the opinion value $o_{i}=0, d_{i}=d_{m}$; when the opinion value $o_{i}=1, d_{i}=d_{n} \cdot d_{m}$ and $d_{n}$ represent the agent's tolerance thresholds when opinion value is 0 and 1 , 
respectively, i.e., $d_{m}$ is the tolerance of the agents with neutral attitudes and lower emotional characteristics and $d_{n}$ is the tolerance of the agents with the extreme opinions. In this paper, we assume that the agent with the extreme opinion has a lower tolerance threshold, i.e., $d_{n}$ is a small positive and constant value $\left(d_{n}=0.001\right)$ and $d_{m}$ is one of the parameters of the simulations $\left(d_{\max }=0.5\right)$. We study the group behavior for different values of $x$. The larger the value of $x$ is, the larger $d_{i}$ is for the same value of $o_{i}$. This indicates that the agents with intermediate opinions present a larger tolerance threshold and are more likely to be influenced by other agents when the value of $x$ is larger. When parameter $x$ is $1 / 2,1$ and 2 , respectively, the graph of function of the tolerance threshold with the opinion value is shown in Figure 1.

As shown in Figure 1, when $o_{i}>o_{j}, d_{i}<d_{j}$, indicating that during the interaction between agents with different opinion values, agent $j$ has a higher tolerance threshold due to the lack of clear opinion intensity, and is accordingly more susceptible to the influence of others. So agent $j$ is easy to change his/her opinion under the impact of agent $i$ during the interaction, while agent $i$ has a clear opinion and lower tolerance threshold, during the interaction with agent $j$, the opinion of agent $j$ is outside the tolerance threshold of agent $i$, so agent $i$ is free from the influence of agent $j$ and keeps his/her initial opinion. Due to the asymmetry of the opinion evolution, the agents with the opinion value close to 0 are more easily affected by the external factors to generate extreme opinions due to his/her larger tolerance threshold, and the agent with extreme opinions is not susceptible to the influence from others due to his/her lower tolerance threshold and keeps his/her initial opinion. Under the impact of this opinion evolution mechanism, every agent may generate extreme opinions, and the network public opinion will eventually form group polarization.

\section{Simulation Results}

3.1. Effects of the Random Distribution of the Initial Opinions. In this paper, we firstly analyze the group opinion evolution under the condition that the initial opinions are randomly distributed. The initial opinions of the agents are randomly distributed in $[0: 1]$, and the globally coupled network in the regular network is used as the basic network. We assume the number of agents in the group is $N=2000$, the agents in the group are fully connected, and any two agents can interact with each other randomly, $\lambda$ follows a normal distribution with a mean value of 0.5 and a standard deviation of 0.2 , the same below. The simulation result in this paper is an average of 200 times. When $x$ is $1 / 2,1$ and 2 , and $d_{m}$ takes different values, the group opinion evolution is shown in Figures 2-4.

Figures 2-4 show the process of the group opinion distribution evolution under different values of $d_{m}$, when $x$ is $1 / 2$, 1 and 2 . The abscissa indicates the opinion values of agents, the ordinates indicate the evolution algebra, and the vertical coordinate indicates the proportion of the number of agents holding same opinion value to the total agent in the group. According to Figures 3 and 4, when $x$ is 1 and 2, respectively, $d_{m}$ takes a smaller value; the various opinion clusters are quickly formed. When the opinion value is close to 1 , the tolerance threshold of agents is the minimum, and the

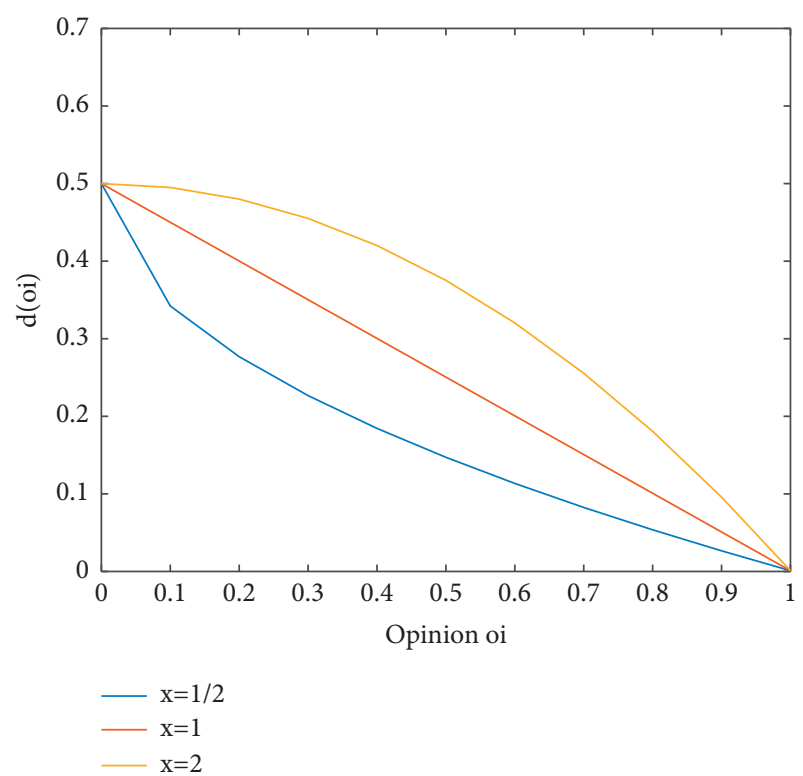

FIGURE 1: Graph of the function of the tolerance threshold $d_{i}$ with the opinion value $o_{i}$, for $x=1 / 2,1,2$ and $d_{\max }=0.5$ and $d_{n}=0.001$. The larger the value of $x$ is, the larger $d_{i}$ is for the same value of $o_{i}$.

proportion of agents around this opinion is also minimal. As the gradual increase of $d_{m}$, the tolerance threshold of the agents in the group gradually gets larger, and the probability of interaction between agents increases. Finally, the agent opinion values are basically close to 1 under the impact of agents with extreme opinions, thus forming group polarization. Compared with the condition when $x=1$, the value of $d_{m}$ is lower when group polarization is formed under $x=2$ (when $x=1$, $d_{m}=0.55$; when $x=2, d_{m}=0.4$ ), which is because the larger tolerance threshold when $x$ takes on a larger value. When $d_{m}$ takes the same value, the average interactive threshold of agents in the group with a large $x$ is greater. Agents are more likely to interact opinions with each other. According to Figure 2, when $x=1 / 2$, the process of the opinion distribution evolution shows a similar tendency as that when $x=1$ and $x=2$. However, after group polarization is formed, $d_{m}$ is at its maximum $\left(d_{m}=0.9\right)$, because the smaller tolerance threshold when $x=1 / 2$ leads to a lower average interactive threshold of agents in the group, thus decreasing the probability of opinion interaction between agents. Only when the tolerance threshold of the agents in the group is large enough, group polarization will be formed under the impact of the extreme opinion.

\subsection{Effects of the Normal Distribution of the Initial Opinions.} For the purpose of studying the process of the group opinion distribution evolution, the initial opinion values of agents are randomly distributed in the above studies. In fact, the initial opinions of the public on events are not randomly distributed in most cases, and the public will express their own opinions on events when it occurs. Generally, the opinions of most of the public on events will be concentrated in the middle region; only minorities will hold the extreme opinions or keep a neutral attitude. As time goes by, the opinions of most agents will gradually become unified under the impact of the minorities with extreme opinions, thus 


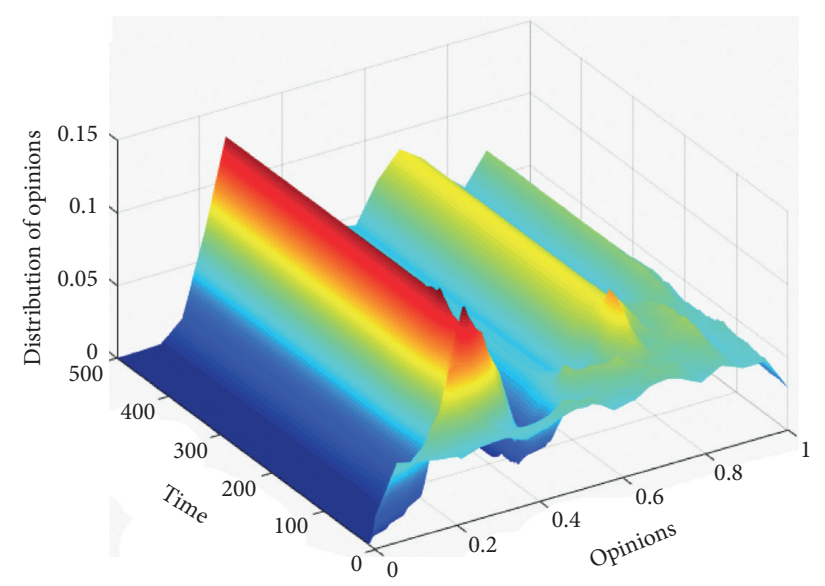

(a)

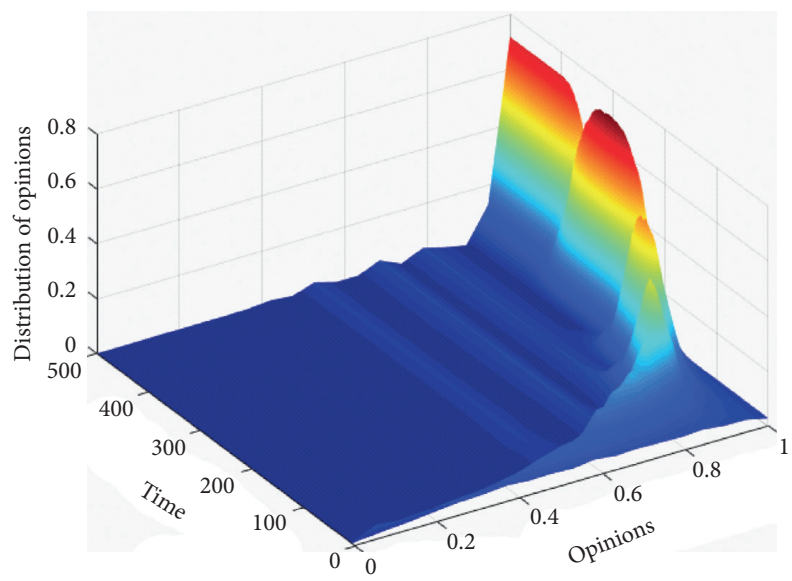

(c)

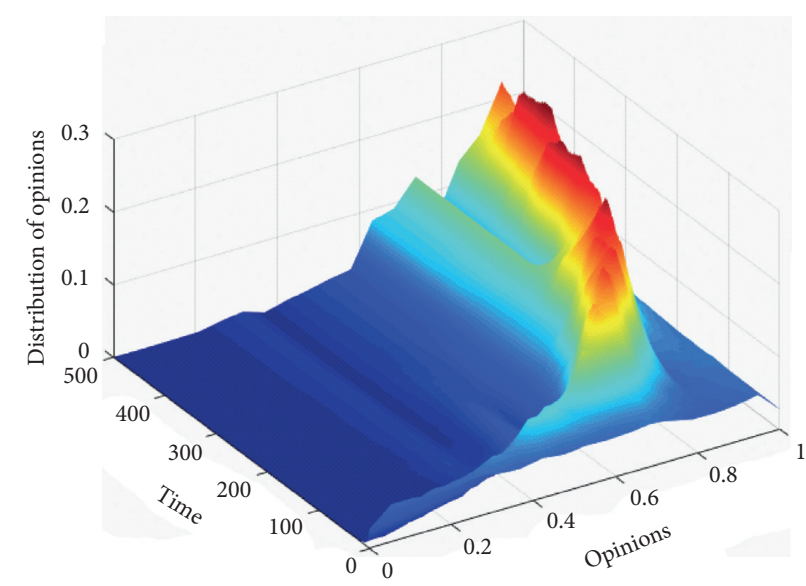

(b)

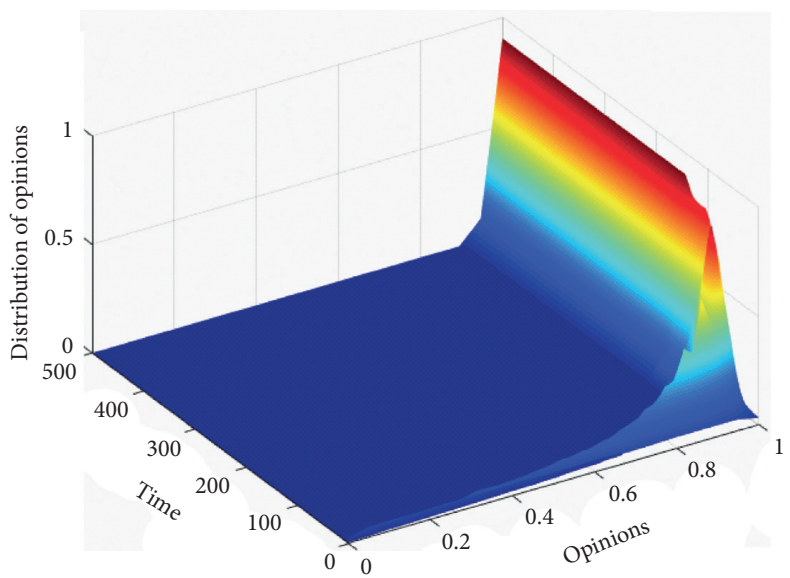

(d)

FIgURE 2: Group opinion distribution evolution when $x=1 / 2$. With the increase of $d_{m}$, the proportion of agents whose opinion value is close to 1 is increasing. (a) $d_{m}=0.25$. (b) $d_{m}=0.5$. (c) $d_{m}=0.65$. (d) $d_{m}=0.9$.

forming group polarization. To study the process of the group opinion distribution evolution under such conditions, we use a normal distribution with a mean value of 0.5 and a standard deviation of 0.2 as the distribution of the initial opinions of agents, in which the initial opinions of the vast majority of agents are between 0 and 1 , while the agents with the opinions close to 0 and 1 account for only a small part. When $x=2$ and $d_{m}$ takes different values, the group opinion evolution is shown in Figure 5.

According to Figure 5, when the initial opinions are normally distributed, group polarization of opinions will be finally formed, with a smaller value of $d_{m}\left(d_{m}=0.3\right)$. When $d_{m}$ takes a small value, there are various opinion clusters concentrated in the middle region, which conforms to the group opinions being normally distributed. With the increase of $d_{m}$, the agent opinions gradually cluster around the opinion value of 1 under the impact of the extreme opinion. Since the opinions are normally distributed, the opinion values of most of the agents are concentrated in the middle region. Compared with the condition under the random distribution of the opinions, the opinion values of most agents are more concentrated, which is more conducive to opinion interaction between agents, so group polarization can be formed when $d_{m}$ takes a small value. This also reflects that in daily life, under the impact of the minority with extreme opinions, the lower proportion of agents with extreme opinions at the early stage will promote the formation of group polarization when most agents have no clear opinion intensity with a higher tolerance threshold of the group, and the higher the proportion of agents with larger tolerance thresholds, the more likely group polarization will be formed.

3.3. Effects of Different Network Structures. Constructing a network model with complex structures to simulate the network relationship in real society is a common way to study the specific social problems. To study the evolution of information dissemination in social networks, scholars have constructed network models with various complex structures, including the regular network, the random network, the small-world network, the BA scale-free network, and others that widely used in researches. In the social network, the information dissemination process is related to the agent characteristics and network structure. To analyze the impacts of different network topology on the information 


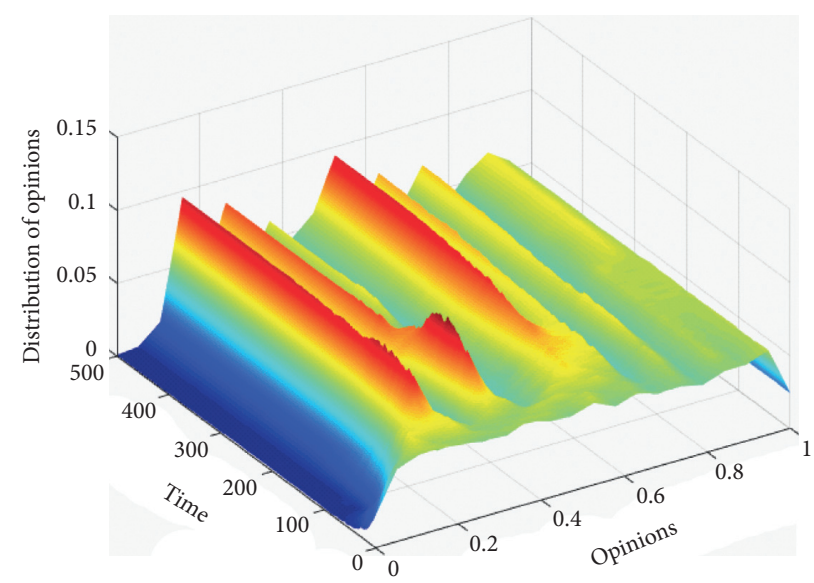

(a)

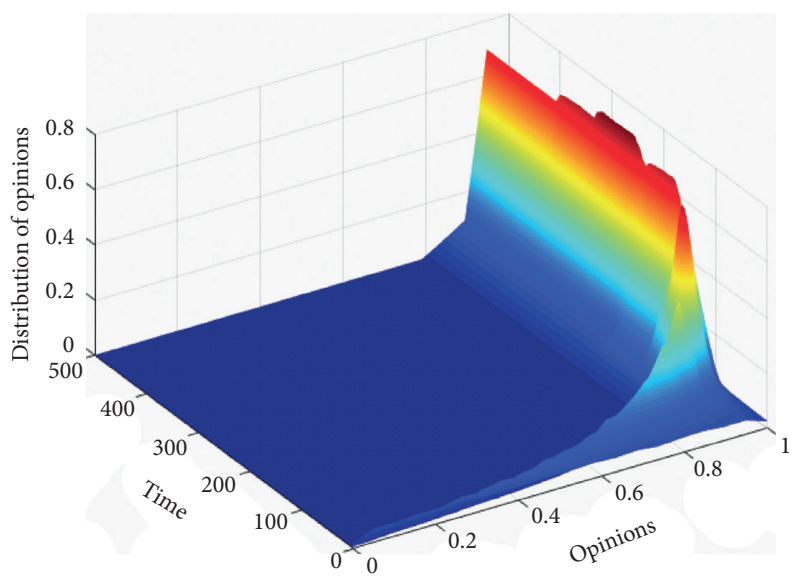

(c)

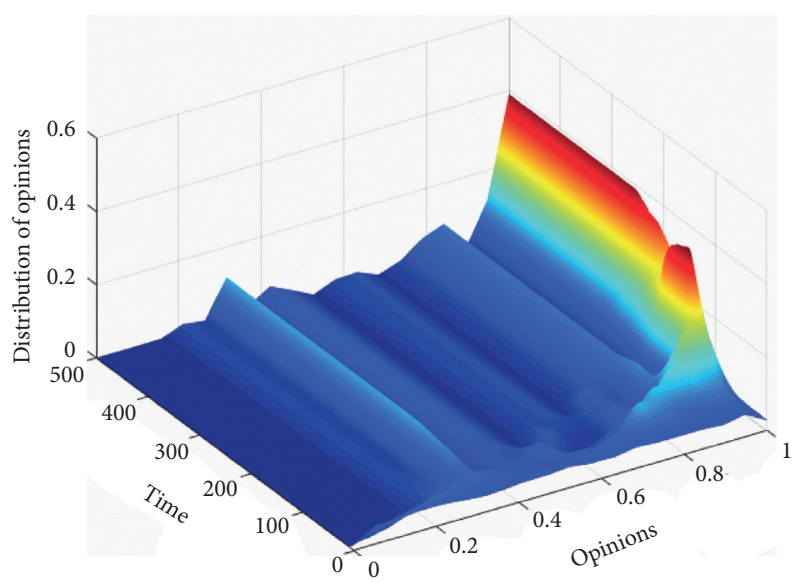

(b)

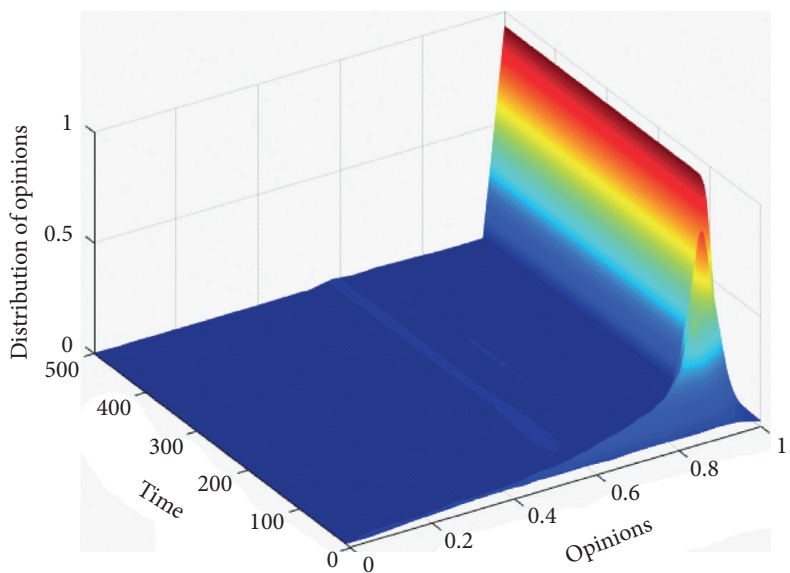

(d)

Figure 3: Group opinion distribution evolution when $x=1$. Compared with the condition when $x=1 / 2$, the value of $d_{m}$ is lower when group polarization is formed under $x=1$. (a) $d_{m}=0.15$. (b) $d_{m}=0.3$. (c) $d_{m}=0.45$. (d) $d_{m}=0.55$.

dissemination process in the social network, we apply the nearest-neighbor coupled network in the regular network, the random network, the small-world network, and the BA scale-free network with the node number of 2000 , respectively. The average degree is set to be consistent based on the construction rules of these networks so that the evolution results of network structures have comparability to the greatest extent. The initial opinions of agents are randomly distributed in [0:1]. When the parameter $x=2$, and $d_{m}$ takes different values, the processes of group opinion distribution evolution in different network structures are shown in Figures 6-9.

According to Figures 6-9, in different network structures, group polarization can be formed under different conditions. When $d_{m}$ takes small value, there are various opinion clusters in these four networks, with the increase of the value of $d_{m}$, group polarization will be finally formed. Meanwhile, when opinion polarization is formed, the values of $d_{m}$ in these four networks are nearest-neighbor coupled network $>$ random network $>$ BA scale-free network $>$ smallworld network. It can be seen from the above analysis, when group polarization is formed in the nearest-neighbor coupled network, the value of $d_{m}$ is at its minimum $\left(d_{m}=0.4\right)$, where the smaller the value of $d_{m}$, the easier it is to form group polarization. When the average tolerance threshold is lower, the consensus of the agent's opinions in the group can be reached, thus forming group polarization. Due to the interaction between agents, the globally coupled network, with same node number, has the maximum average degree and clustering coefficients and the minimum average path length, so it is most likely to form group polarization. The small-world network has larger clustering coefficients and a shorter average path length, which increases the probability of interactions between different agents in the group, thus promoting the interaction between agents. The clustering coefficients of BA scale-free network and regular network are lower than that of the small-world network, and the degree of interaction between agents is also lower than that of small-world network. However, due to "priority connection," one of the characteristics of BA scale-free network, there are a few nodes with larger scale, which connect with a large number of nodes, increasing the probability of interaction between other agents and the agent to achieve a full interaction between agents, so the BA scale-free networks are more likely to form opinion polarization than the regular networks. In the nearest-neighbor coupled network, the 


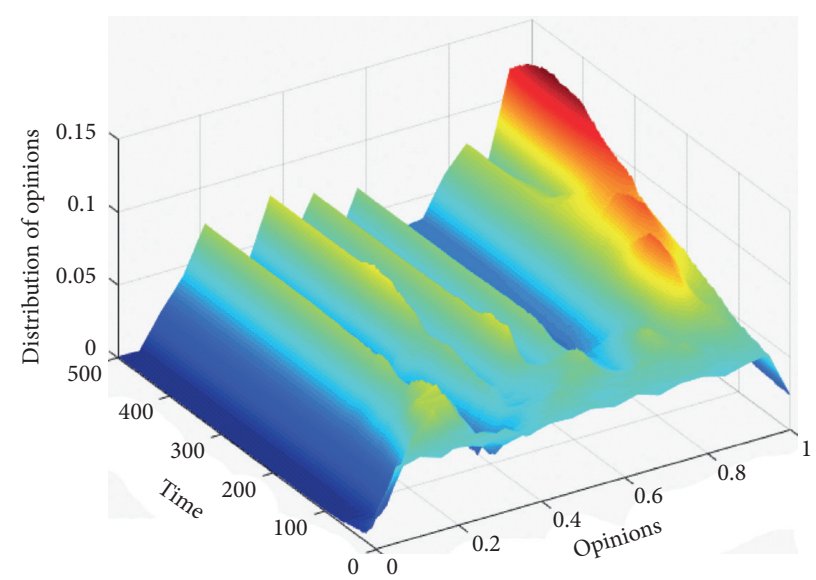

(a)

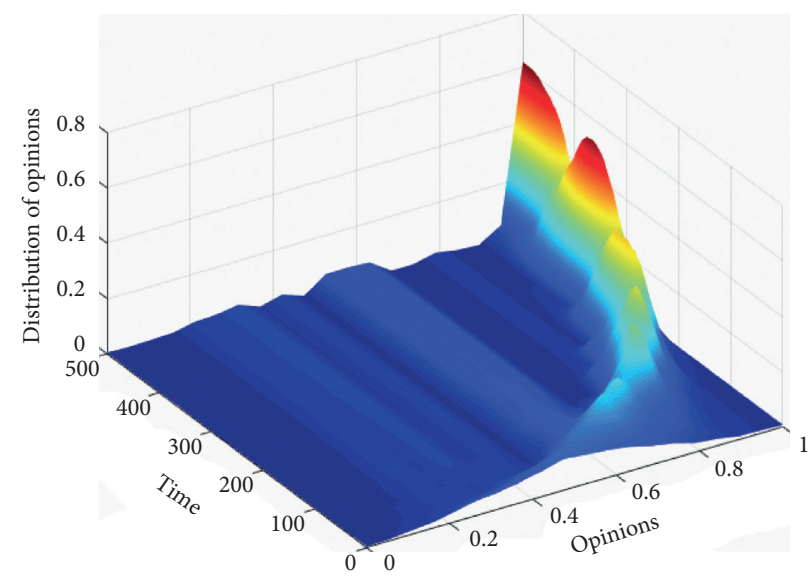

(c)

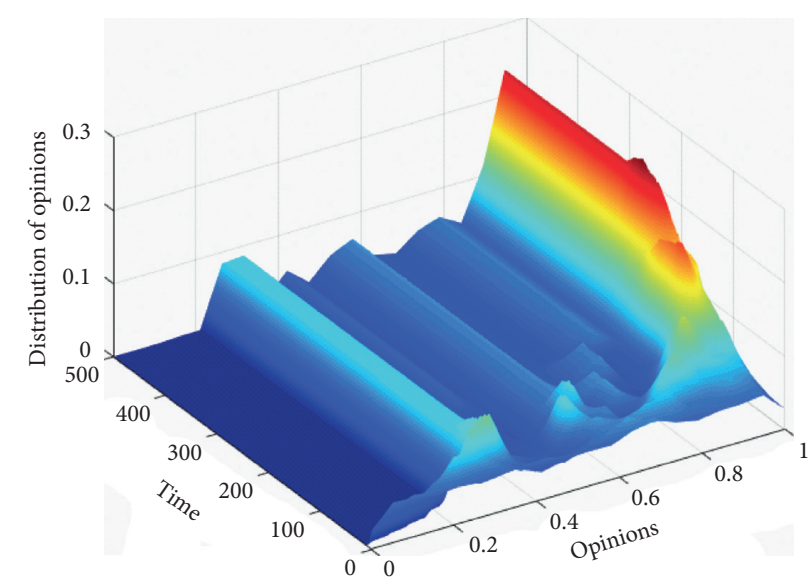

(b)

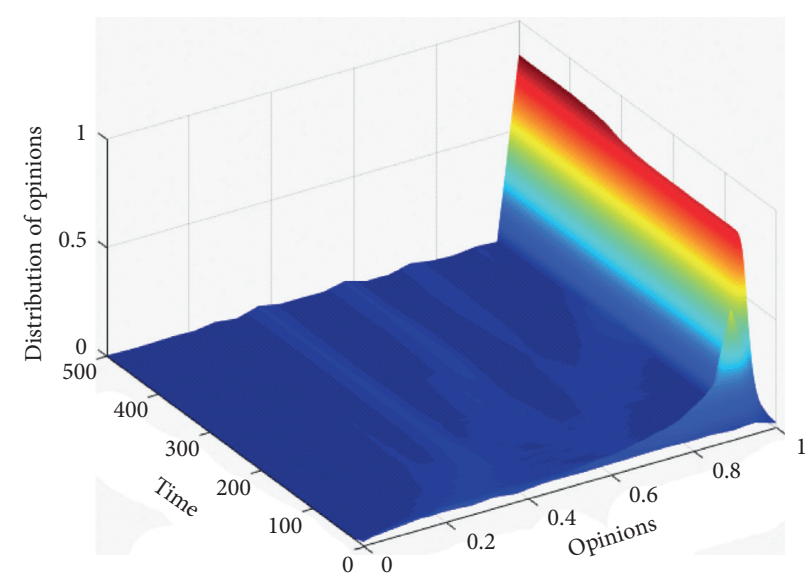

(d)

FIGURE 4: Group opinion distribution evolution when $x=2$. Compared with the conditions when $x=1 / 2$ and $x=1$, the value of $d_{m}$ is lower when group polarization is formed under $x=2$. (a) $d_{m}=0.1$. (b) $d_{m}=0.2$. (c) $d_{m}=0.3$. (d) $d_{m}=0.4$.

agents only interact with their adjacent agents. Although its larger clustering coefficient can promote the interaction between agents, its larger average path length will confine the interaction between agents to different local areas, making opinion clusters not able to interact with each other, so the value of $d_{m}$ is at its maximum when group polarization is formed.

3.4. Effects of Opinion Leaders. Opinion leaders, as influential public figures, play an important role in the development of the network public opinion. Generally, they will affect the opinions of the public and lead the development direction of the network public opinion, thus promoting group polarization. Due to the "priority connection" characteristic of scale-free network, there are a few nodes with a larger degree, which accounts for a small proportion of the total number of nodes in the group, but these nodes connect with a large number of other nodes that play a significant role in the whole network. In this paper, we take the BA scale-free network as the basic network, the initial opinions of agents are randomly distributed in [0: 1], and two nodes with the largest degree are selected as the "opinion leaders."
Due to the stronger professional competence and knowledge reserve, opinion leaders always firmly insist on their opinion and are less likely to be influenced by the external factors to change their opinions, so the opinion acceptance of the opinion leaders is set as $\lambda \in[0,0.01]$. Similarly, the range of agent's opinion is extended to $[-1: 1]$, among which, $[-1: 0]$ and $[0: 1]$ indicate the disagreement and agreement toward the event, respectively, and two nodes with larger scale are selected to make their opinion values within the two opinion intervals, respectively. When $x=2$, and $d_{m}$ takes different values, the processes of group opinion distribution evolution are shown in Figures 10 and 11.

In Figure 10, two nodes with the largest degree are selected as the opinion leaders, with the initial opinion values of the opinion leaders are 0.5 . When $d_{m}=0.45$, there are two opinion clusters in the group, among which, the opinion value of the opinion cluster with the larger agent proportion is close to 0.5 , while the other is close to 0.8 ; when $d_{m}=0.9$, there is only one opinion cluster in the group, the opinion value of the opinion cluster is close to 0.8 . In Figure 4 , when $d_{m}=0.45$, group polarization has been formed, with the opinion value close to 1 , indicating the opinion leaders hinder the formation of group polarization in a certain 


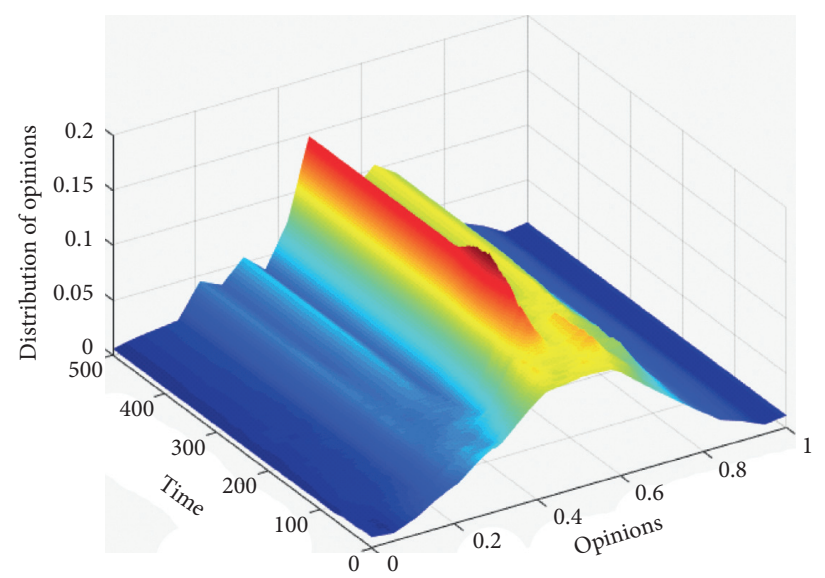

(a)

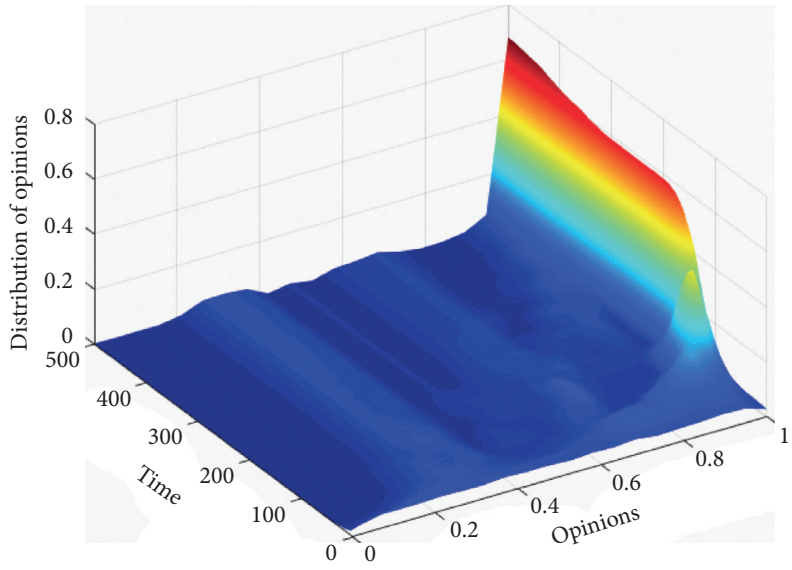

(c)

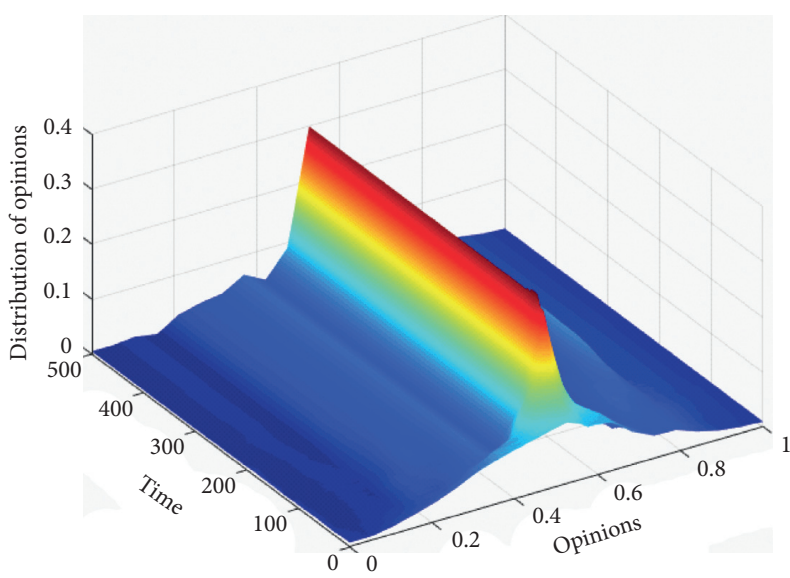

(b)

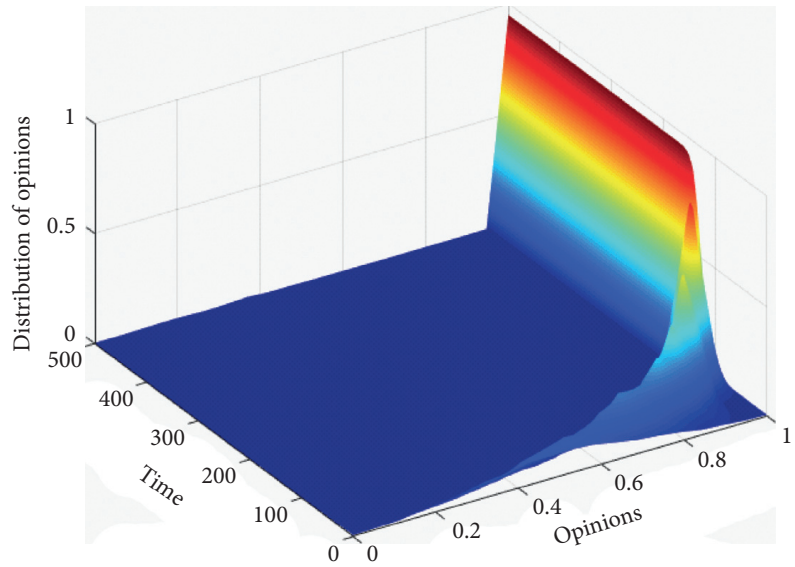

(d)

FIGURE 5: Group opinion distribution evolution under the normal distribution of the initial opinions. Compared with the random distribution of the initial opinions, the value of $d_{m}$ is lower when group polarization is formed under the normal distribution of the initial opinions. (a) $d_{m}=0.1$. (b) $d_{m}=0.15$. (c) $d_{m}=0.2$. (d) $d_{m}=0.3$.

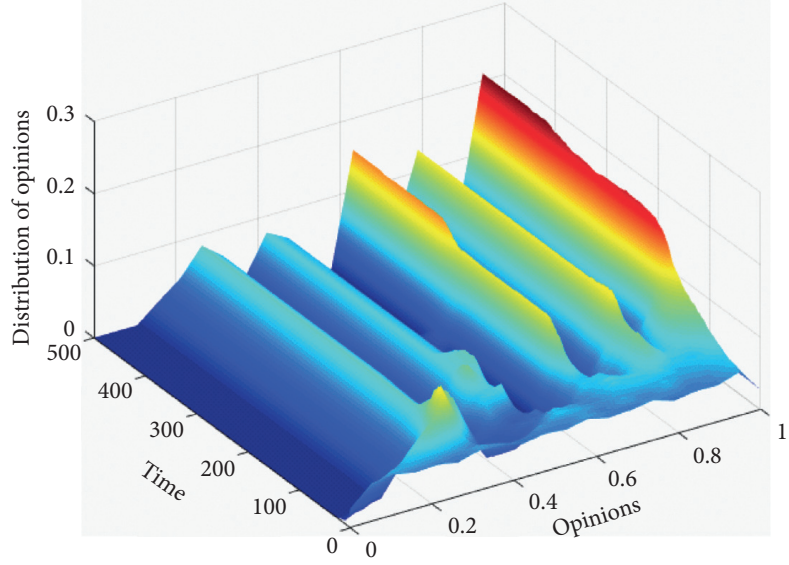

(a)

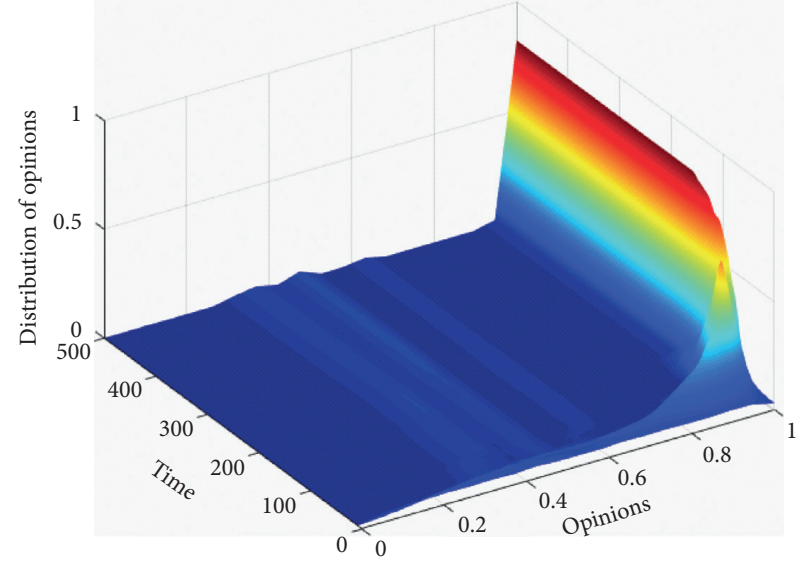

(b)

FIGURE 6: Group opinion distribution evolution in the nearest-neighbor coupled network. Group polarization is formed when $d_{m}=0.65$. (a) $d_{m}=0.3$. (b) $d_{m}=0.65$. 


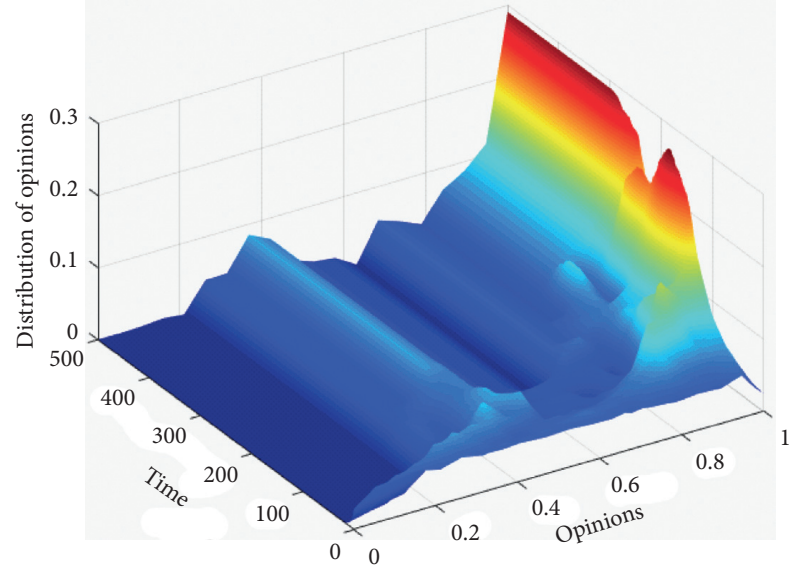

(a)

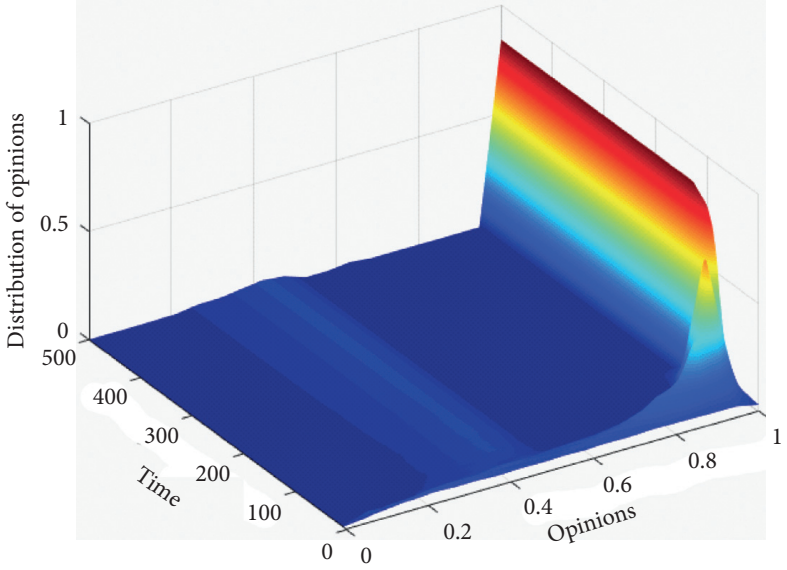

(b)

FIGURE 7: Group opinion distribution evolution in the random network. Group polarization is formed when $d_{m}=0.6$. (a) $d_{m}=0.3$. (b) $d_{m}=0.6$.

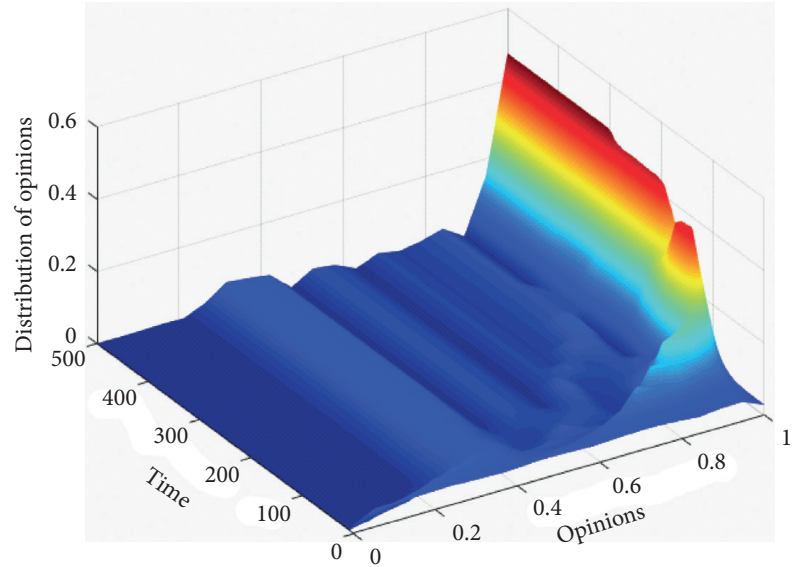

(a)

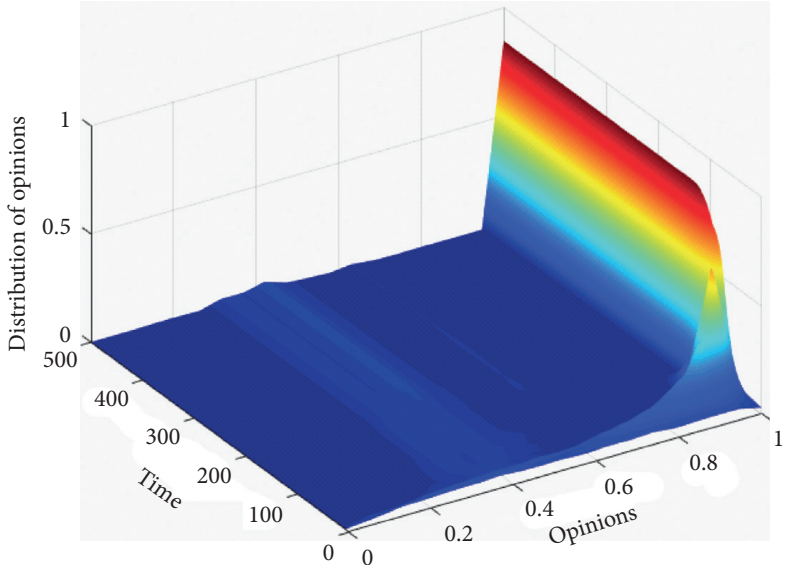

(b)

FigURE 8: Group opinion distribution evolution in the small-world network. Group polarization is formed when $d_{m}=0.52$. (a) $d_{m}=0.3$. (b) $d_{m}=0.52$.

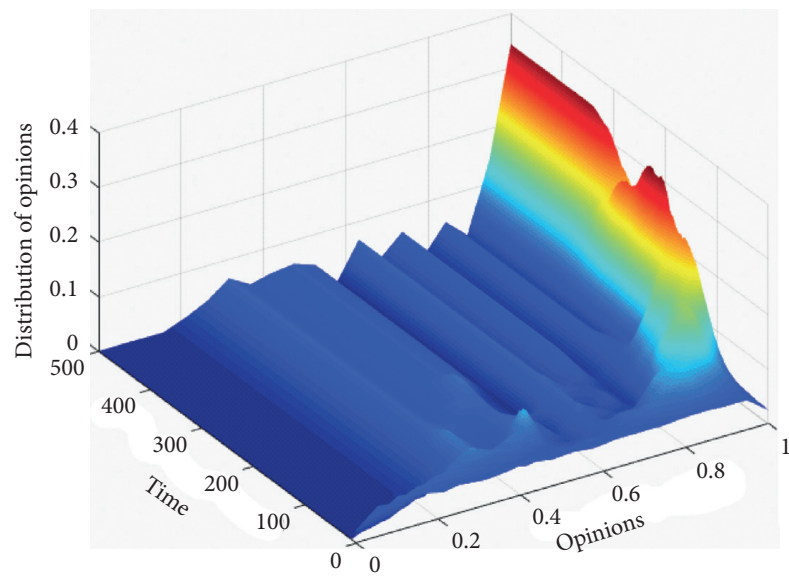

(a)

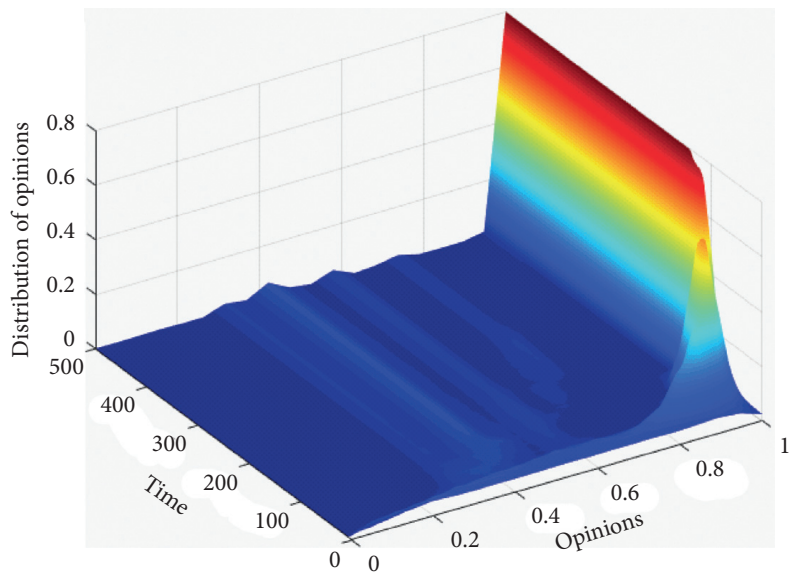

(b)

FIGURE 9: Group opinion distribution evolution in the BA scale-free network. Group polarization is formed when $d_{m}=0.58$. (a) $d_{m}=0.3$. (b) $d_{m}=0.58$. 


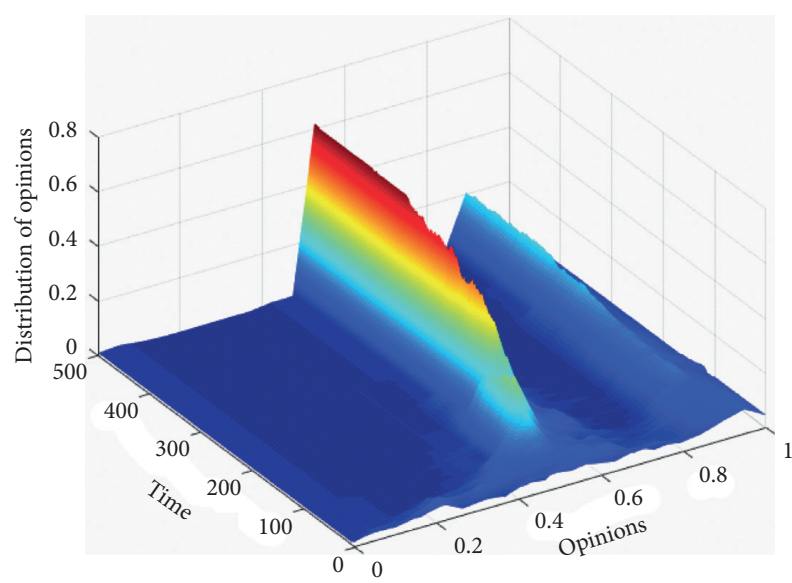

(a)

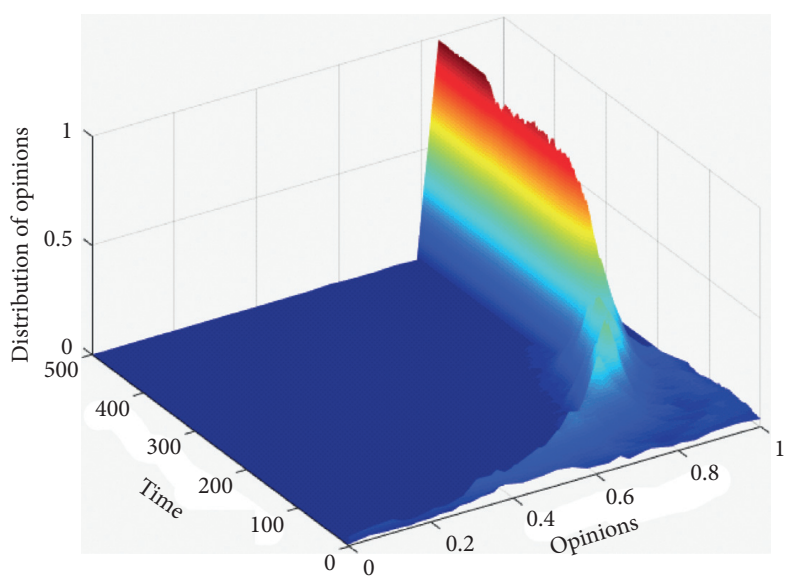

(b)

Figure 10: Two opinion leaders with positive opinion values. There are two opinion clusters when $d_{m}=0.45$, the opinion value of the opinion cluster with the larger agent proportion is close to 0.5 , while the other is close to 0.8 ; there is only one opinion cluster when $d_{m}=0.9$, the opinion value of the opinion cluster is close to 0.8. (a) $d_{m}=0.45$. (b) $d_{m}=0.9$.

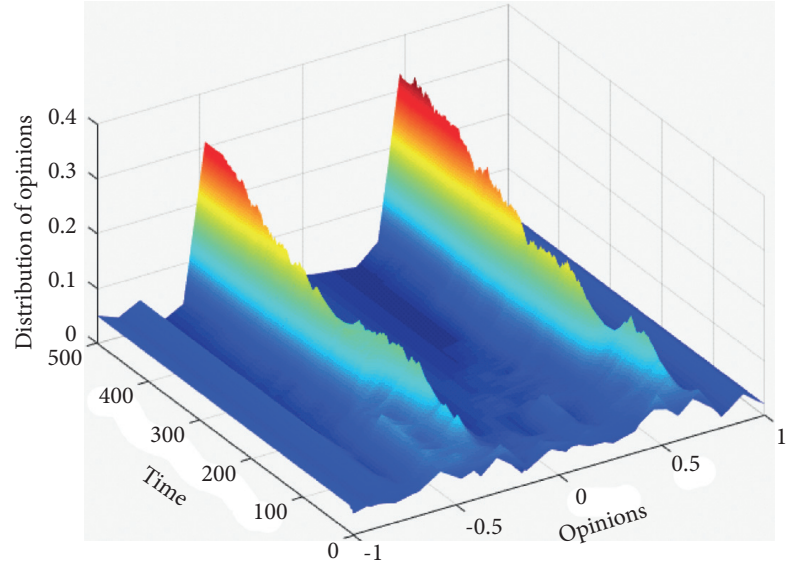

(a)

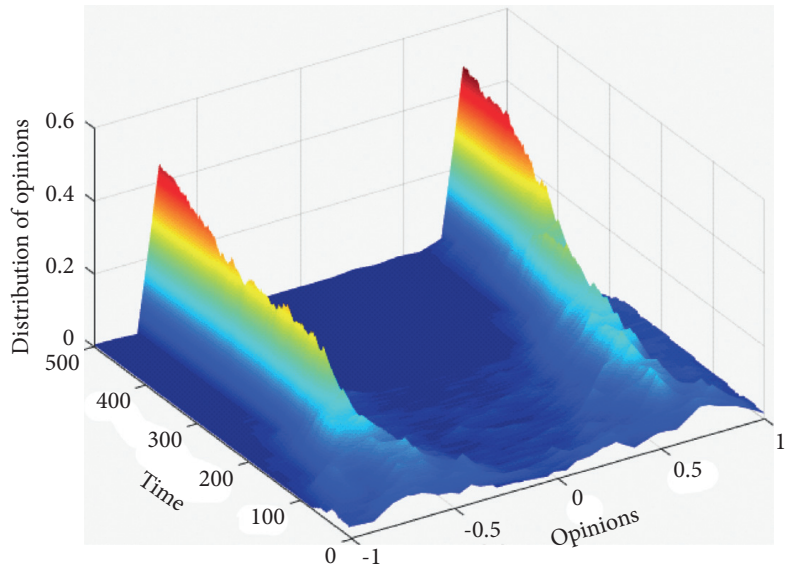

(b)

Figure 11: Two opinion leaders with opposite opinion values. There are two opinion clusters when $d_{m}=0.45$; the opinion values of these two clusters are close to 0.5 and -0.5 , respectively; there are two opinion clusters when $d_{m}=0.9$; the opinion values of these two clusters are close to 0.8 and -0.8 , respectively. (a) $d_{m}=0.45$. (b) $d_{m}=0.9$.

extent. When $d_{m}$ takes a smaller value, agents in the group will be impacted by the opinion leaders, forming the opinion cluster with the similar opinion with the opinion leaders. With the increase of $d_{m}$, agents in the group have a higher tolerance threshold, and the group opinion will gradually close to the opinion value of 1 under the impact of the agents with extreme opinions. However, combined with the impact of opinion leaders, when group polarization is finally formed, $d_{m}$ takes a higher value, and finally, the opinion value is around 0.8. In Figure 11, two nodes with the largest degree are selected as the opinion leaders, with the initial opinion values of the opinion leaders are 0.5 and -0.5 , respectively. When $d_{m}=0.45$, there are two opinion clusters with larger agent proportion of the group, and the opinion values of these two clusters are close to 0.5 and -0.5 , respectively. When $d_{m}=0.9$, group polarization is formed near the endpoint values of the two opinion intervals, indicating that the opinion values of other agents in the group will move toward the opinion value of the two opinion leaders, and the opinion values of the finally formed opinion polarization are near the 0.8 and -0.8 , respectively.

\section{Conclusion}

In this paper, we construct an opinion evolution model with agent emotional characteristics and credibility based on the extended Deffuant model in the bounded confidence models. We make full use of the theory of opinion interval of the RA model to connect the agent firmness with opinion values through agent emotional characteristics and credibility. The model constructed in this paper qualitatively interprets the reason for group polarization and considers the heterogeneity of agents in reality. In addition, in order to quantitatively analyze group polarization, in reality, we 
consider the different initial opinion distribution and several ordinary complex network structures and the impact of opinion leaders on the group opinion evolution.

The results show that compared with that under the random distribution of the initial opinions, group polarization is formed under the normal distribution of initial opinions, and the value of $d_{m}$ is smaller. Under the impact of the minority with extreme opinions, the lower proportion of agents with extreme opinions at the early stage will promote the formation of group polarization when the most agents have no clear opinion intensity with a higher tolerance threshold of the group, and the higher the proportion of agents with larger tolerance thresholds, the more likely group polarization will be formed. Different network structures have different impacts on the group opinion evolution. The larger clustering coefficients and shorter average path length are more likely to facilitate opinion interactions between agents in a group. Since any two agents in the globally coupled network can interact with each other, it has the largest clustering coefficients and the shortest average patch length, so it is more likely to achieve group polarization. Relying on the "priority connection" characteristic of the BA scale-free network, a large number of nodes in the network conduct the opinion interaction with the node at a higher probability; when the tolerance threshold is lower, group polarization will be formed. Opinion leaders will impact the group opinion evolution. When $d_{m}$ takes a small value, the agents in the group will be affected by the opinion leaders and form the opinion cluster with similar opinion values as that of opinion leaders. Meanwhile, when group polarization is formed, the value of $d_{m}$ is larger.

According to the results, we can find that the variable parameter $d_{m}$ plays an important role in the group opinion evolution. The larger the $d_{m}$ is, the larger the average tolerance threshold of the agents in the group, the higher the proportion of the agents with a larger tolerance threshold, which is more conducive to opinion interaction among agents. Meanwhile, under the effect of the agents with extreme opinions, the unified opinions are gradually formed, thus forming group polarization. However, the agents with extreme opinions have strong emotional characteristics, lower tolerance threshold, and are not susceptible to the external factors, so it is difficult to change the opinion of these agents with extreme opinions. We can decrease the probability of polarization by lowering the tolerance threshold of the group. For example, at the early stage of the event, governments can guide the public to form clear opinion intensity and fully play the roles of the mainstream media and opinion leaders in the guidance process, thus making the network public opinion develops toward a better direction. The application of social software makes the connections between agents with the structural characters of small-world network and BA scale-free network, which promote group polarization. Therefore, the government should strengthen the supervision of the information dissemination in social software. In addition, regulator can increase the cost of manipulating the public opinion by increasing the punishment for the malicious manipulation behavior, so as to reduce the manipulation behavior of opinion leaders.
The conclusions of the paper provide a certain theoretical foundation for interpreting the reasons for group polarization in our daily life and put forward corresponding countermeasures to cope with the vicious group polarization and to play the positive effect of group polarization. However, there are many shortcomings in this paper. The opinion interaction between agents, in reality, depends on whether they are connected or not, and the strength of this connection relationship also has a significant impact on the tolerance between agents. Therefore, the group opinion evolution based on weighted network structures is more realistic. In addition, the network structures are varied over time. The impact of the network structure with dynamic characteristics on the group opinion evolution is the next research focus.

\section{Data Availability}

No data were used in the study.

\section{Disclosure}

Shuyang Jiang and Hu Wang are co-first authors.

\section{Conflicts of Interest}

The authors declare that they have no conflicts of interest.

\section{Authors' Contributions}

Shuyang Jiang and $\mathrm{Hu}$ Wang contributed equally to this work.

\section{Acknowledgments}

This research was supported by the Fundamental Research Funds for the Central Universities and Scientific Research Foundation of Graduate School of Southeast University (YBPY2146).

\section{References}

[1] A. Fang, K. Yuan, J. Geng, and X. Wei, "Opinion dynamics with bayesian learning," Complexity, vol. 2020, Article ID 8261392, 5 pages, 2020.

[2] G. Olivares, J. P. Cárdenas, J. C. Losada, and J. Borondo, "Opinion polarization during a dichotomous electoral process," Complexity, vol. 2019, Article ID 5854037, 9 pages, 2019.

[3] C. Castellano, S. Fortunato, and V. Loreto, "Statistical physics of social dynamics," Reviews of Modern Physics, vol. 81, no. 2, pp. 591-646, 2009.

[4] J. Wu, J. Chang, Q. Cao, and C. Liang, "A trust propagation and collaborative filtering based method for incomplete information in social network group decision making with type2 linguistic trust," Computers \& Industrial Engineering, vol. 127, pp. 853-864, 2019.

[5] X. Chen, Z. Wu, H. Wang, and W. Li, "Impact of heterogeneity on opinion dynamics: heterogeneous interaction model," Complexity, vol. 2017, Article ID 5802182, 10 pages, 2017.

[6] M. Cao, J. Wu, F. Chiclana, R. Ureña, and E. Herrera-Viedma, "A personalized feedback mechanism based on maximum 
harmony degree for consensus in group decision making," IEEE Transactions on Systems, Man and Cybernetics: Systems, 2019.

[7] M. Perc, "The social physics collective," Scientific Reports, vol. 9, no. 1, p. 16549, 2019.

[8] D. Helbing, D. Brockmann, T. Chadefaux et al., "Saving human lives: what complexity science and information systems can contribute," Journal of Statistical Physics, vol. 158, no. 3, pp. 735-781, 2015.

[9] S. Galam, "Rational group decision making: a random field ising model at $T=0$," Physica A: Statistical Mechanics and its Applications, vol. 238, no. 1-4, pp. 66-80, 1997.

[10] M. Mobilia, "Does a single zealot affect an infinite group of voters?” Physical Review Letters, vol. 91, no. 2, Article ID 028701, 2003.

[11] M. Mobilia, A. Petersen, and S. Redner, "On the role of zealotry in the voter model," Journal of Statistical Mechanics: Theory and Experiment, vol. 8, pp. 266-276, 2007.

[12] S. Galam and F. Jacobs, "The role of inflexible minorities in the breaking of democratic opinion dynamics," Physica A: Statistical Mechanics and its Applications, vol. 381, pp. 366-376, 2007.

[13] S. Galam, "Minority opinion spreading in random geometry," The European Physical Journal B, vol. 25, no. 4, pp. 403-406, 2002.

[14] K. Sznajd-Weron, M. Tabiszewski, and A. M. Timpanaro, "Phase transition in the sznajd model with independence," EPL (Europhysics Letters), vol. 96, no. 4, p. 48002, 2011.

[15] A. Benatti, H. F. de Arruda, F. N. Silva, C. H. Comin, and L. da Fontoura Costa, "Opinion diversity and social bubbles in adaptive sznajd networks," Journal of Statistical Mechanics: Theory and Experiment, vol. 2020, no. 2, Article ID 023407, 2020.

[16] G. Deffuant, "Comparing extremism propagation patterns in continuous opinion models," Journal of Artificial Societies and Social Simulation, vol. 9, no. 3, pp. 1-8, 2006.

[17] P. Sobkowicz, "Extremism without extremists: deffuant model with emotions," Frontiers in Physics, vol. 3, p. 17, 2015.

[18] R. Ureña, G. Kou, Y. Dong, F. Chiclana, and E. HerreraViedma, "A review on trust propagation and opinion dynamics in social networks and group decision making frameworks," Information Sciences, vol. 478, pp. 461-475, 2019.

[19] A. Woolcock, C. Connaughton, Y. Merali, and F. Vazquez, "Fitness voter model: damped oscillations and anomalous consensus," Physical Review E, vol. 96, no. 3, Article ID 032313, 2017.

[20] E. Hashemi, M. Pirani, A. Khajepour, B. Fidan, A. Kasaiezadeh, and S.-K. Chen, "Opinion dynamics-based vehicle velocity estimation and diagnosis," IEEE Transactions on Intelligent Transportation Systems, vol. 19, no. 7, pp. 2142-2148, 2017.

[21] P. Frasca, S. Tarbouriech, and L. Zaccarian, "Hybrid models of opinion dynamics with opinion-dependent connectivity," Automatica, vol. 100, pp. 153-161, 2019.

[22] Y. Dong, Y. Fan, H. Liang, F. Chiclana, and E. HerreraViedma, "Preference evolution with deceptive interactions and heterogeneous trust in bounded confidence model: a simulation analysis," Knowledge-Based Systems, vol. 175, pp. 87-95, 2019.

[23] C. Altafini and F. Ceragioli, "Signed bounded confidence models for opinion dynamics," Automatica, vol. 93, pp. 114-125, 2018.
[24] Z. Cheng, Y. Xiong, and Y. Xu, “An opinion diffusion model with decision-making groups: the influence of the opinion's acceptability," Physica A: Statistical Mechanics and its Applications, vol. 461, pp. 429-438, 2016.

[25] K. Li, H. Liang, G. Kou, and Y. Dong, "Opinion dynamics model based on the cognitive dissonance: an agent-based simulation," Information Fusion, vol. 56, pp. 1-14, 2020.

[26] K. Burghardt, W. Rand, and M. Girvan, "Competing opinions and stubborness: connecting models to data," Physical Review E, vol. 93, no. 3, Article ID 032305, 2016.

[27] A. Jędrzejewski and K. Sznajd-Weron, "Impact of memory on opinion dynamics," Physica A: Statistical Mechanics and its Applications, vol. 505, pp. 306-315, 2018.

[28] W.Su, G. Chen, and Y. Hong, "Noise leads to quasi-consensus of Hegselmann-Krause opinion dynamics," Automatica, vol. 85, pp. 448-454, 2017.

[29] F. Amblard and G. Deffuant, "The role of network topology on extremism propagation with the relative agreement opinion dynamics," Physica A: Statistical Mechanics and its Applications, vol. 343, pp. 725-738, 2004.

[30] J. Ghaderi and R. Srikant, "Opinion dynamics in social networks with stubborn agents: equilibrium and convergence rate," Automatica, vol. 50, no. 12, pp. 3209-3215, 2014.

[31] P. Walla, G. Brenner, and M. Koller, "Objective measures of emotion related to brand attitude: a new way to quantify emotion-related aspects relevant to marketing," PLoS One, vol. 6, no. 11, Article ID e26782, 2011.

[32] M. Del Vicario, G. Vivaldo, A. Bessi et al., "Echo chambers: emotional contagion and group polarization on facebook," Scientific Reports, vol. 6, no. 1, pp. 1-12, 2016.

[33] Y. Kim and Y. Kim, "Incivility on facebook and political polarization: the mediating role of seeking further comments and negative emotion," Computers in Human Behavior, vol. 99, pp. 219-227, 2019.

[34] G. Deffuant, F. Amblard, G. Weisbuch, and T. Faure, "How can extremism prevail? a study based on the relative agreement interaction model," Journal of Artificial Societies and Social Simulation, vol. 5, no. 4, 2002.

[35] G. Deffuant, D. Neau, F. Amblard, and G. Weisbuch, "Mixing beliefs among interacting agents," Advances in Complex Systems, vol. 3, no. 01n04, pp. 87-98, 2000.

[36] G. Deffuant, F. Amblard, and G. Weisbuch, "Modelling group opinion shift to extreme: the smooth bounded confidence model," 2004, https://arxiv.org/abs/0410199.

[37] M. J. Brandt, A. M. Evans, and J. T. Crawford, "The unthinking or confident extremist? political extremists are more likely than moderates to reject experimenter-generated anchors," Psychological Science, vol. 26, no. 2, pp. 189-202, 2015.

[38] G. Weisbuch, G. Deffuant, F. Amblard, and J.-P. Nadal, "Interacting agents and continuous opinions dynamics," in Heterogenous Agents, Interactions and Economic Performance, pp. 225-242, Springer, Berlin, Germany, 2003. 